Website: ijetms.in Issue:4, Volume No.4, July-2020 DOI: 10.46647/ijetms.2020.v04i04.005

\title{
Basic Components of Web Services Architecture: A Survey
}

\author{
${ }^{1}$ M. Kiran Kumar, ${ }^{2}$ VJST Anirudh \\ ${ }^{1}$ Assistant professor, Department of Information Technology \\ Guru Nanak Institutions Technical Campus, Hyderabad-Telangana \\ ${ }^{1}$ Under Graduate, Guru Nanak Institutions Technical Campus, Hyderabad \\ Kirann.intell@gmail.com \\ anirudhvjst@gmail.com
}

\begin{abstract}
Service provided by one electronic device to the other electronic device or devices is generally called as a web service. It may be a block of code that can be remotely accessed and utilized. In the present IT industry web services are playing a vital role in application development by providing a facility of using the existing modules to make up an application without coding from the scratch. This improves the productivity and reduces the overall project development cost and time. This paper provides the basic information of the components that supports the usage of web service along with the web service architecture. The components that are required for the web service architecture such as SOAP: simple object access protocol: used for establishing connection between components, UDDI (Universal Description, Discovery, and Integration):a registry that stores all the web services and WSDL: web service description language.
\end{abstract}

Keywords: Web Services, SOAP, WSDL and UDDI.

INTRODUCTION: The service provided by a device which is fully equipped and capable, to the other systems in the cluster or group over internet is called as a web service. Web service mainly reduces the risk of writing code from the scratch during application development which reduces the overall cost and time of the entire project.

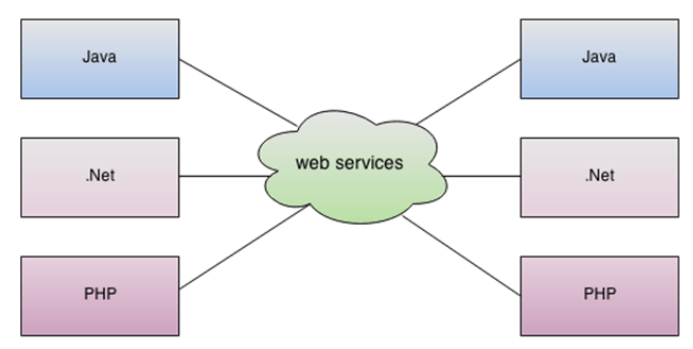

Fig: 1-Web Service

In the figure, one application can communicate with other applications through web service over the network. suppose .net application can .net or PHP applications in unique way of communication, here that language is called web service.

There are two types of web services.

1. SOAP web services

2. RESTful web services

\section{SOAP Web service}

SOAP stands for Simple Object Access protocol. To communicate between 2 parties XML based protocols are used which uses SOAP envelope. It also provides additional security with its Web Service security.

\section{Restful web service}

Rest stands for Representational state transfer. Unlike SOAP which is protocol based Restful web service are architectural based services. In Rest architecture, everything is treated as resource and which is identified by unique URI. It can handle any type of data which could be XML, JSON or anything.

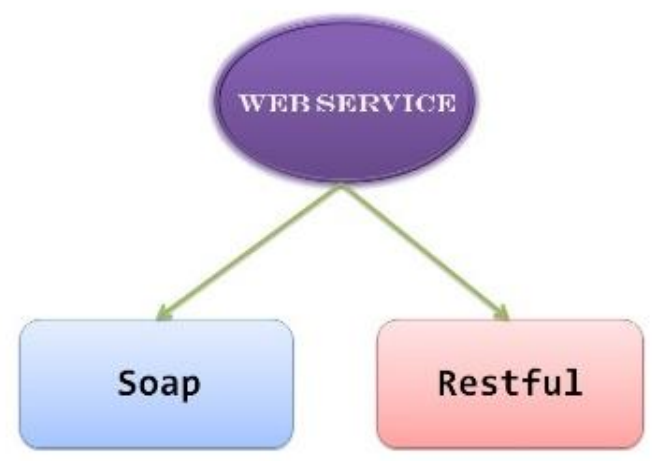




\section{International Journal of Engineering Technology and Management Sciences[IJETVIS]}

Website: ijetms.in Issue:4, Volume No.4, July-2020 DOI: 10.46647/ijetms.2020.v04i04.005

Fig: 2- Web services categories

\section{Emergence of Web Services:}

Today, the intensity of Internet and empowering Internet-based applications has made a universe of discrete and assortment business applications, which coincide in a similar innovation space however without interfacing with one another. The increasing demands of the industry for enablingB2B, application-to application [1] [2]. By adopting Web technologies, the serviceoriented facilitates and the delivery of services over the Internet by leveraging standard technologies such as XML[1]. It uses platform-neutral standards by exposing the underlying application components and making them available to any application, any platform, or any device, and at any location. Today, this phenomenon is well adopted for implementation and is commonly referred to as Web services.

\section{ARCHITECTURE:}

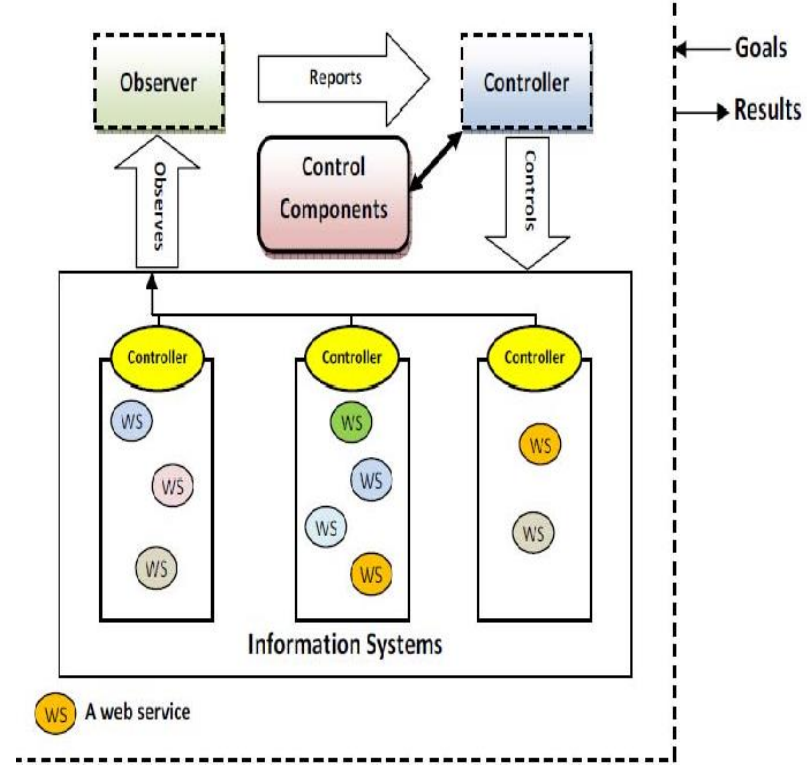

SOAP

Fig: 3-Architecture

SOAP is known as the Simple Object Access Protocol and was developed by Develop Mentor, Inc., as a platform independent protocol for accessing services, objects between applications, and servers using HTTP-based communication. SOAP uses the
XML based vocabulary and the return types. It was developed as an intermediate language so that applications built on various programming languages could talk easily to each other and avoid the extreme development effort.

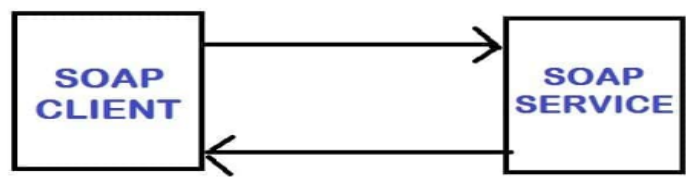

\section{Advantages of SOAP}

It is the perfect medium which was developed in order to achieve a common language for the web services to talk to their clients [2].

It is a lightweight protocol which is used in the data exchange. It is platform independent and operating system independent.

SOAP Message Structure SOAP messages are auto generated by web services when it is called.

SOAP Message has the following elements The Envelope element, the header element The body element, the Fault element

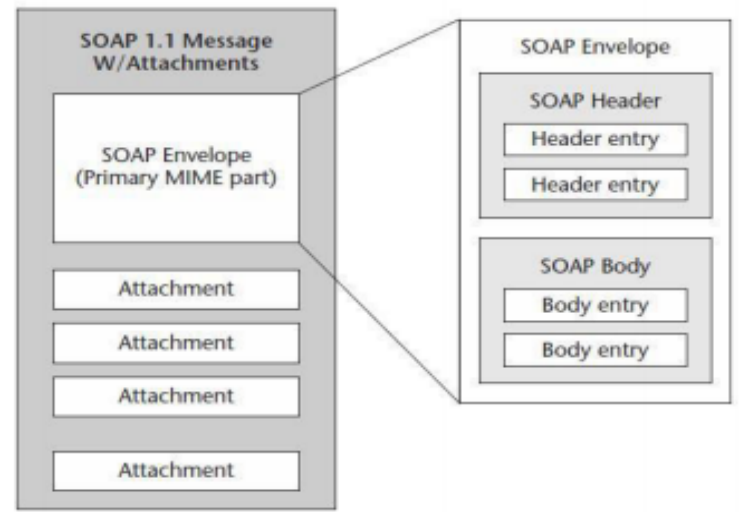

Fig: 5 Structure of SOAP

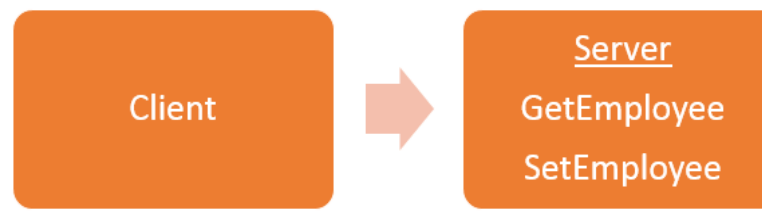

Fig-6 SOAP communication model

WSDL 
Website: ijetms.in Issue:4, Volume No.4, July-2020 DOI: 10.46647/ijetms.2020.v04i04.005

WSDL is abbreviated as Web Services Description Language. WSDL was developed jointly by Microsoft and IBM. It's a standard format to describe a web service. WSDL is the combination of XML schema and SOAP over the internet to provide various web services [3].

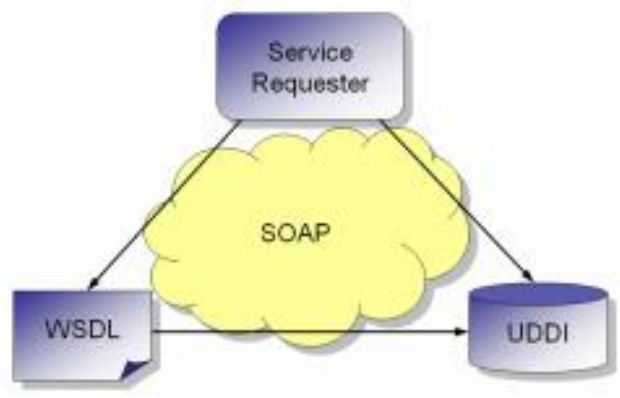

Fig:7-SOAP as Intermediate

It is a XML-based convention utilized in the trading of data decentralized and appropriated situations. It portrays how to get to a web administration and what activities it will perform. It is a fundamental piece of UDDI. It will break down web services into three specific, identifiable elements that can be combined or reused once it is defined.

The three major elements of WSDL are:-

- Types

- Operations

- Binding

To see the value of WSDL, imagine you want to start calling a SOAP method provided by one of your business partners. You could ask him for some sample SOAP messages and write your application to produce and consume messages that look like the samples, but this can be error prone. For example, you might see a customer ID of 4889 and assume it's an integer when in fact it's a string. WSDL specifies what a request message containand what the response message will look like in unambiguous notation.WSDL must will become the best way to author SOAP interfaces for XML Web services. UDDI
UDDI was found by Microsoft, IBM, and Ariba. Universal Discovery Description and Integration is the yellow pages of Web services. It is a platform independent markup language-based registry by which on the internet the business can be listed and it a process to register and locate the web service applications.

A UDDI registry consists of components like white pages and yellow pages where each has its own features. White pages represents the address and contact information of the service provider and the yellow pages maintains the details like categories of that particular service.

It also consists of green pages which maintain the full technical details of the service.

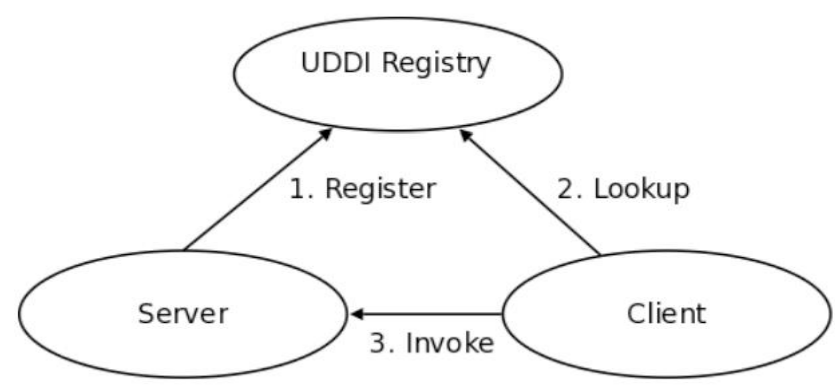

Fig :8- UDDI Registry

UDDI architecture

Data model

API specification

Cloud services

UDDI Data Model

UDDI includes an XML Schema that describes four five data structures:

- businessEntity

- businessService

- bindingTemplate

- tModel

\section{CONCLUSION:}

The basic information of The components that are required for the web service architecture such as SOAP: simple object access protocol: used for establishing connection between components, UDDI (Universal Description, Discovery, and 
Website: ijetms.in Issue:4, Volume No.4, July-2020 DOI: 10.46647/ijetms.2020.v04i04.005

Integration):a registry that stores all the web services and WSDL: web service description language.

\section{REFERENCES:}

[1]M. Kiran Kumar, S. Jessica Saritha. AN EFFICIENT APPROACH TO QUERY REFORMULATION IN WEB SEARCH, International Journal of Research in Engineering and Technology. 2015;p.172.

[2] Bhargavi, $K \&$ Kumar, $M$ \& Hussan, M.I. \& Stalin, D. (2020). HS2Cloud: A Secure Lightweight Framework for Image Storage on Hybrid Cloud.

[3]https://mrcet.com/downloads/digital_notes/CS E/IV\%20Year/WEB\%20SERVICES_17.11.2018.p $d f$ 